\title{
REACHABILITY OF CONE FRACTIONAL CONTINUOUS-TIME LINEAR SYSTEMS
}

\author{
TADEUSZ KACZOREK
}

\author{
Faculty of Electrical Engineering \\ Białystok Technical University, ul. Wiejska 45D, 15-351 Białystok, Poland \\ e-mail: kaczorek@isep.pw.edu.pl
}

\begin{abstract}
A new class of cone fractional continuous-time linear systems is introduced. Necessary and sufficient conditions for a fractional linear system to be a cone fractional one are established. Sufficient conditions for the reachability of cone fractional systems are given. The discussion is illustrated with an example of linear cone fractional systems.
\end{abstract}

Keywords: cone fractional system, linear systems, reachability.

\section{Introduction}

In positive systems inputs, state variables and outputs take only non-negative values. Examples of positive systems are industrial processes involving chemical reactors, heat exchangers and distillation columns, storage systems, compartmental systems, water and atmospheric pollution models. A variety of models having positive linear systems behaviour can be found in engineering, management science, economics, social sciences, biology and medicine, etc.

Positive linear systems are defined on cones and not on linear spaces. Therefore, the theory of positive systems is more complicated and less advanced. An overview of state of the art in positive systems is given in the monographs (Farina and Rinaldi, 2000; Kaczorek, 2002). An extension of positive systems are cone systems. The notion of cone systems was introduced in (Kaczorek, 2006). Roughly speaking, a cone system is a system obtained from a positive one by the substitution of the positive orthants of states, inputs and outputs by suitable arbitrary cones. The realization problem for cone systems was addressed in (Kaczorek, 2006; Kaczorek, 2007c).

The first definition of the fractional derivative was introduced by Liouville and Rieman at the end of the 19th century (Miller and Ross, 1993; Nishimoto, 1984; Podlubny, 1999). This idea was used by engineers for modelling various processes in the late 1960s (Vinagre et al., 1960; Vinagre and Feliu, 2002; Zaborowsky and Meylaov, 2001). Mathematical fundamentals of fractional calculus are given in the monographs (Miller and Ross, 1993;
Nishimoto, 1984; Oldham and Spanier, 1974; Oustaloup, 1995; Podlubny, 1999). Fractional order controllers were developed in (Oustaloup, 1993; Podlubny et al., 1997). A generalization of the Kalman filter for fractional order systems was proposed in (Sierociuk and Dzieliński, 2007). Some other applications of fractional order systems can be found in (Engheta, 1997; Ferreira and Machado, 2003; Ostalczyk, 2000; Ostalczyk, 2004; Ostalczyk, 2004; Reyes-Melo et al., 2004; Riu et al., 2001; Samko et al., 1993; Sjöberg and Kari, 2002; Vinagre and Feliu, 2002).

In (Ortigueira, 1997), a method was set forth for the computation of the impulse responses from the frequency responses for fractional standard (non-positive) discrete-time linear systems. Fractional polynomials and $n D$ systems were investigated in (Gałkowski, 2005). Necessary and sufficient conditions for the reachability and controllability to zero of cone fractional discrete-time linear systems were established in (Kaczorek, 2007a; Kaczorek, 2007b; Kaczorek, 2007d).

In this paper, the notion of cone fractional linear continuous-time systems will be introduced. Sufficient conditions for the reachability of cone fractional linear systems will be established. To the best of the author's knowledge, cone fractional continuous-time linear systems have not been considered yet.

\section{Positive fractional linear systems}

Let $\mathbb{R}^{n \times m}$ be the set of $n \times m$ real matrices and $\mathbb{R}^{n}:=$ $\mathbb{R}^{n \times 1}$. The set of $m \times n$ real matrices with nonnegative 
entries will be denoted by $\mathbb{R}_{+}^{m \times n}$ and $\mathbb{R}_{+}^{n}:=\mathbb{R}_{+}^{n \times 1}$. The set of nonnegative integers will be denoted by $\mathbb{Z}_{+}$and the $n \times n$ identity matrix by $I_{n}$.

The following Caputo definition of the fractional derivative will be used (Miller and Ross, 1993; Podlubny, 1999):

$$
\begin{aligned}
D^{\alpha} f(t)= & \frac{\mathrm{d}^{\alpha}}{\mathrm{d} t^{\alpha}} f(t) \\
= & \frac{1}{\Gamma(k-\alpha)} \int_{0}^{t} \frac{f^{(k)}(\tau)}{(t-\tau)^{\alpha+1-k}} \mathrm{~d} \tau, \\
& \quad k-1<\alpha \leq k \in \mathbb{N}=\{1,2, \ldots\},
\end{aligned}
$$

where $\alpha \in \mathbb{R}$ is the order of the fractional derivative and $f^{(k)}(\tau)=\mathrm{d}^{k} f(\tau) / \mathrm{d} \tau^{k}$. Consider the continuous-time fractional linear system described by the state equation

$$
\begin{aligned}
\frac{\mathrm{d}^{\alpha}}{\mathrm{d} t^{\alpha}} x(t) & =A x(t)+B u(t), \quad 0<\alpha \leq 1, \\
y(t) & =C x(t)+D u(t),
\end{aligned}
$$

where $x(t) \in \mathbb{R}^{n}, u(t) \in \mathbb{R}^{m}, y(t) \in \mathbb{R}^{p}$ are respectively the state, input and output vectors, and $A \in \mathbb{R}^{n \times n}, B \in$ $\mathbb{R}^{n \times m}, C \in \mathbb{R}^{p \times n}, D \in \mathbb{R}^{p \times m}$

Theorem 1. The solution of Eqn. (2a) is given by

$$
x(t)=\Phi_{0}(t) x_{0}+\int_{0}^{t} \Phi(t-\tau) B u(t) \mathrm{d} \tau, \quad x(0)=x_{0},
$$

where

$$
\begin{aligned}
\Phi_{0}(t) & =E_{\alpha}\left(A t^{\alpha}\right)=\sum_{k=0}^{\infty} \frac{A^{k} t^{k \alpha}}{\Gamma(k \alpha+1)}, \\
\Phi(t) & =\sum_{k=0}^{\infty} \frac{A^{k} t^{(k+1) \alpha-1}}{\Gamma[(k+1) \alpha]},
\end{aligned}
$$

and $E_{\alpha}\left(A t^{\alpha}\right)$ is the Mittag-Leffler matrix function. What is more,

$$
\Gamma(x)=\int_{0}^{\infty} e^{-t} t^{x-1} \mathrm{~d} t
$$

is the Gamma function.

Definition 1. The system (2) is called the internally positive fractional system if and only if $x(t) \in \mathbb{R}_{+}^{n}$ and $y(t) \in \mathbb{R}_{+}^{p}, t \geq 0$ for any initial conditions $x_{0} \in \mathbb{R}_{+}^{n}$ and all inputs $u(t) \in \mathbb{R}_{+}^{m}, t \geq 0$.

A square rational matrix $A=\left[a_{i j}\right]$ is called a Metzler matrix if its off-diagonal entries are nonnegative, i.e., $a_{i j} \geq 0$ for $i \neq j$ (Farina and Rinaldi, 2000; Kaczorek, 2002). The set of $n \times n$ all Metzler matrices will be denoted by $M_{n}$.

Theorem 2. The continuous-time fractional system (2) is internally positive if and only if

$$
A \in M_{n}, B \in \mathbb{R}_{+}^{n \times m}, C \in \mathbb{R}_{+}^{p \times n}, \quad D \in \mathbb{R}_{+}^{p \times m} .
$$

\section{Cone fractional systems}

Based on (Kaczorek, 2008), the following definitions are recalled.

\section{Definition 2. Let}

$$
P=\left[\begin{array}{c}
p_{1} \\
\vdots \\
p_{n}
\end{array}\right] \in \mathbb{R}^{n \times n}
$$

be nonsingular and $p_{k}$ be its $k$-th row $(k=1, \ldots, n)$. The set

$$
\mathcal{P}:=\left\{x \in \mathbb{R}^{n}: \bigcap_{k=1}^{n} p_{k} x \geq 0\right\}
$$

is called a linear cone generated by the matrix $P$. In a similar way, for the inputs $u$, we may define the linear cone

$$
\mathcal{Q}:=\left\{u \in \mathbb{R}^{m}: \bigcap_{k=1}^{m} q_{k} u \geq 0\right\}
$$

generated by the nonsingular matrix

$$
Q=\left[\begin{array}{c}
q_{1} \\
\vdots \\
q_{m}
\end{array}\right] \in \mathbb{R}^{m \times m}
$$

and for the outputs $y$, the linear cone

$$
\mathcal{V}:=\left\{y \in \mathbb{R}^{p}: \bigcap_{k=1}^{p} v_{k} y \geq 0\right\}
$$

generated by the nonsingular matrix

$$
V=\left[\begin{array}{c}
v_{1} \\
\vdots \\
v_{p}
\end{array}\right] \in \mathbb{R}^{p \times p} .
$$

Definition 3. The fractional system (2) is called a $(\mathcal{P}, \mathcal{Q}, \mathcal{V})$ cone fractional system if $x(t) \in \mathcal{P}$ and $y(t) \in \mathcal{V}$, $t \geq 0$ for every $x_{0} \in \mathcal{P}, u(t) \in \mathcal{Q}, t \geq 0$.

The $(\mathcal{P}, \mathcal{Q}, \mathcal{V})$ cone fractional system (2) will be briefly called the cone fractional system. If $\mathcal{P}=\mathbb{R}_{+}^{n}$, $\mathcal{Q}=\mathbb{R}_{+}^{m}, \mathcal{V}=\mathbb{R}_{+}^{n}$, then the $\left(\mathbb{R}_{+}^{n},{ }_{-} \mathbb{R}_{+}^{m}, \mathbb{R}_{+}^{p}\right)$ cone system is equivalent to the classical positive system (Farina and Rinaldi, 2000; Kaczorek, 2002).

Theorem 3. The fractional system (2) is a $(\mathcal{P}, \mathcal{Q}, \mathcal{V})$ cone fractional one if and only if

$$
\begin{array}{ll}
\bar{A}=P A P^{-1} \in M_{n}, & \bar{B}=P B Q^{-1} \in \mathbb{R}_{+}^{n \times m}, \\
\bar{C}=V C P^{-1} \in \mathbb{R}_{+}^{p \times n}, & \bar{D}=V D Q^{-1} \in \mathbb{R}_{+}^{p \times m} .
\end{array}
$$


Proof. Let

$$
\begin{aligned}
& \bar{x}(t)=P x(t), \quad \bar{u}(t)=Q u(t), \\
& \bar{y}(t)=V y(t), \quad t \geq 0 .
\end{aligned}
$$

From Definition 3 it follows that if $x(t) \in \mathcal{P}$, then $\bar{x}(t) \in$ $\mathbb{R}_{+}^{n}$, if $u(t) \in \mathcal{Q}$, then $\bar{u}(t) \in \mathbb{R}_{+}^{m}$, and if $y(t) \in \mathcal{V}$, then $\bar{y}(t) \in \mathbb{R}_{+}^{p}$. From (2) and (11) we have

$$
\begin{aligned}
\frac{\mathrm{d}^{\alpha} \bar{x}(t)}{\mathrm{d} t^{\alpha}} & =\frac{\mathrm{d}^{\alpha} P x(t)}{\mathrm{d} t^{\alpha}}=P A x(t)+P B u(t) \\
& =P A P^{-1} \bar{x}(t)+P B Q^{-1} \bar{u}(t) \\
& =\bar{A} \bar{x}(t)+\bar{B} \bar{u}(t), \quad t \geq 0
\end{aligned}
$$

and

$$
\begin{aligned}
\bar{y}(t) & =V y(t)=V C x(t)+V D u(t) \\
& =V C P^{-1} \bar{x}(t)+V D Q^{-1} \bar{u}(t) \\
& =\bar{C} \bar{x}(t)+\bar{D} \bar{u}(t), \quad t \geq 0 .
\end{aligned}
$$

It is well known (Kaczorek, 2002; Kaczorek, 2007b) that the system (12) is positive if and only if the conditions (10) are satisfied.

\section{Reachability}

\subsection{Positive fractional systems.}

Definition 4. The state $x_{f} \in \mathbb{R}_{+}^{n}$ of the positive fractional system (2) is called reachable in time $t_{f}$ if there exists an input $u(t) \in \mathbb{R}_{+}^{m}, t \in\left[0, t_{f}\right]$ which steers the state of the system (2) from the zero initial state $x_{0}=0$ to the state $x_{f}$. If each state $x_{f} \in \mathbb{R}_{+}^{n}$ is reachable in time $t_{f}$, then the system is called reachable in time $t_{f}$. If for each state $x_{f} \in \mathbb{R}_{+}^{n}$ there exists a time $t_{f}$ such that the state is reachable in time $t_{f}$, then the system (2) is called reachable.

A real square matrix is called monomial if and only if each of its rows and columns contains only one positive entry and the remaining entries are zero.

Theorem 4. (Kaczorek, 2008) The positive fractional system (2) is reachable in time $t_{f}$ if the matrix

$$
R\left(t_{f}\right)=\int_{0}^{t_{f}} \Phi(\tau) B B^{T} \Phi^{T}(\tau) \mathrm{d} \tau
$$

is monomial. The input which steers the state of (2) from $x_{0}=0$ to $x_{f}$ is given by

$$
u(t)=B^{T} \Phi^{T}\left(t_{f}-t\right) R^{-1}\left(t_{f}\right) x_{f},
$$

where ' $T$ ' denotes the transpose.

\subsection{Cone fractional systems.}

Definition 5. A state $x_{f} \in \mathcal{P}$ of the cone fractional system (2) is called reachable in time $t_{f}$ if there exists an input sequence $u(t) \in \mathcal{Q}, t \in\left[0, t_{f}\right]$ which steers the state of the system from the zero initial state $x_{0}=0$ to the desired state $x_{f}$, i.e., $x\left(t_{f}\right)=x_{f}$. If each state $x_{f} \in \mathcal{P}$ is reachable in time $t_{f}$, then the cone fractional system is called reachable in time $t_{f}$. If for every state $x_{f} \in \mathcal{P}$ there exists a time $t_{f}$ such that the state is reachable in time $t_{f}$, then the cone fractional system is called reachable.

Theorem 5. The cone fractional system (2) is reachable in time $t_{f}$ if the matrix

$$
\bar{R}\left(t_{f}\right)=P \int_{0}^{t_{f}} \Phi(\tau) B Q^{-1} Q^{-T} B^{T} \Phi^{T}(\tau) \mathrm{d} \tau P^{T}
$$

here $\left(Q^{-T}=\left(Q^{-1}\right)^{T}\right)$ is a monomial matrix.

Proof. From the relations (11) it follows that if $x(t) \in \mathcal{P}$, then $\bar{x}(t)=\operatorname{Px}(t) \in \mathbb{R}_{+}^{n}, t \geq 0$, and if $u(t) \in \mathcal{Q}$, then $\bar{u}(t)=Q u(t) \in \mathbb{R}_{+}^{m}, t \geq 0$. Hence, by Definitions 4 and 5 , the cone fractional system (2) is reachable in time $t_{f}$ if the positive fractional system (12) is reachable in time $t_{f}$.

From (10) and (5) we have

$$
\begin{aligned}
\bar{\Phi}(t) & =\sum_{k=0}^{\infty} \frac{\bar{A}^{k} t^{(k+1) \alpha-1}}{\Gamma[(k+1) \alpha]} \\
& =\sum_{k=0}^{\infty} \frac{\left(P A P^{-1}\right)^{k} t^{(k+1) \alpha-1}}{\Gamma[(k+1) \alpha]}=P \Phi(t) P^{-1}
\end{aligned}
$$

since $\bar{A}^{k}=\left(P A P^{-1}\right)^{k}=P A^{k} P^{-1}$ for $k=1,2, \ldots$ and

$$
\bar{\Phi}(t) \bar{B}=P \Phi(t) P^{-1} P B Q^{-1}=P \Phi(t) B Q^{-1} .
$$

Using (13) and (17), we obtain

$$
\begin{aligned}
\bar{R}\left(t_{f}\right) & =\int_{0}^{t_{f}} \bar{\Phi}(\tau) \bar{B} \bar{B}^{T} \bar{\Phi}^{T}(\tau) \mathrm{d} \tau \\
& =\int_{0}^{t_{f}}\left(P \Phi(\tau) B Q^{-1}\right)\left(P \Phi(\tau) B Q^{-1}\right)^{T} \mathrm{~d} \tau \\
& =P \int_{0}^{t_{f}} \Phi(\tau) B Q^{-1} Q^{-T} B^{T} \Phi^{T}(\tau) \mathrm{d} \tau P^{T}
\end{aligned}
$$

Therefore, by Theorem 4, the positive fractional system (12) and the cone fractional system (2) are reachable in time $t_{f}$ if the matrix (15) is monomial.

By Theorem 5 we have the following result.

Corollary 1. If $Q=I_{m}$, then $\bar{R}\left(t_{f}\right)=P R\left(t_{f}\right) P^{T}$, and the cone fractional system (2) is reachable in time $t_{f}$ if the positive fractional system is reachable and $P$ is a monomial matrix. 


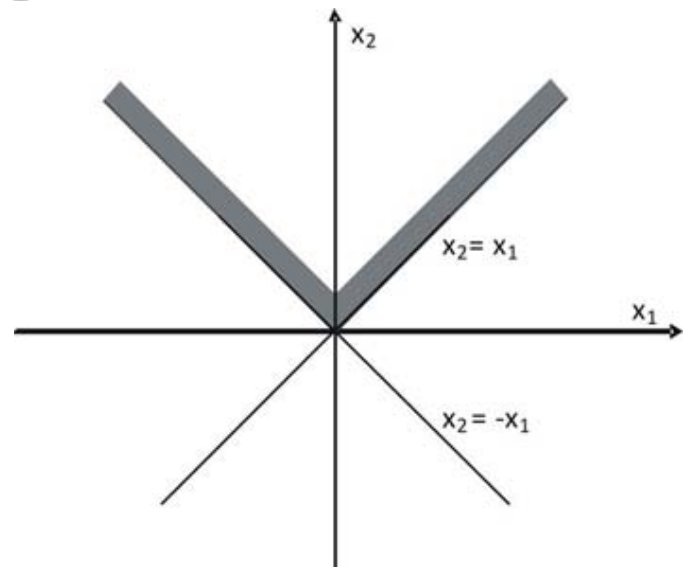

Fig. 1. $\mathcal{P}$-cone generated by the matrix $P$.

Example 1. Consider the cone fractional system (2) with

$$
\begin{aligned}
& P=\left[\begin{array}{cc}
1 & 1 \\
-1 & 1
\end{array}\right], \quad Q=\left[\begin{array}{ll}
1 & 0 \\
0 & 1
\end{array}\right], \\
& A=\left[\begin{array}{ll}
1 & 0 \\
0 & 0
\end{array}\right], \quad B=\left[\begin{array}{ll}
0 & 1 \\
1 & 0
\end{array}\right] .
\end{aligned}
$$

The $\mathcal{P}$-cone generated by the matrix $P$ is shown in Fig. 1 .

In (Kaczorek, 2007d), it was shown that

$$
\Phi(t) B=\left[\begin{array}{cc}
0 & \Phi_{1}(t) \\
\Phi_{2}(t) & 0
\end{array}\right]
$$

and

$$
\begin{aligned}
R\left(t_{f}\right) & =\int_{0}^{t_{f}} \Phi(\tau) B B^{T} \Phi^{T}(\tau) \mathrm{d} \tau \\
& =\int_{0}^{t_{f}}\left[\begin{array}{cc}
\Phi_{1}^{2}(\tau) & 0 \\
0 & \Phi_{2}^{2}(\tau)
\end{array}\right] \mathrm{d} \tau
\end{aligned}
$$

where

$$
\Phi_{1}(t)=\sum_{k=0}^{\infty} \frac{t^{(k+1) \alpha-1}}{\Gamma[(k+1) \alpha]}, \quad \Phi_{2}(t)=\frac{t^{\alpha-1}}{\Gamma(\alpha)}
$$

for $0<\alpha<1$. The matrix (21) is monomial and by Theorem 4 the positive fractional system is reachable in time $t_{f}$.

In case $Q=I_{2}$, the matrix

$$
\begin{aligned}
& \bar{R}\left(t_{f}\right) \\
& =P R\left(t_{f}\right) P^{T} \\
& =\int_{0}^{t_{f}}\left[\begin{array}{cc}
1 & 1 \\
-1 & 1
\end{array}\right]\left[\begin{array}{cc}
\Phi_{1}^{2}(\tau) & 0 \\
0 & \Phi_{2}^{2}(\tau)
\end{array}\right]\left[\begin{array}{cc}
1 & -1 \\
1 & 1
\end{array}\right] \mathrm{d} \tau \\
& =\int_{0}^{t_{f}}\left[\begin{array}{cc}
\Phi_{1}^{2}(\tau)+\Phi_{2}^{2}(\tau) & \Phi_{2}^{2}(\tau)-\Phi_{1}^{2}(\tau) \\
\Phi_{2}^{2}(\tau)-\Phi_{1}^{2}(\tau) & \Phi_{1}^{2}(\tau)+\Phi_{2}^{2}(\tau)
\end{array}\right] \mathrm{d} \tau
\end{aligned}
$$

is not monomial since $\Phi_{1}^{2}(t) \neq \Phi_{2}^{2}(t)$. Therefore, the sufficient condition of Theorem 5 for the reachability in time $t_{f}$ is not satisfied.

From this example and the comparison of (13) and (15), it follows that the sufficient conditions for the reachability of cone fractional systems is much stronger than for positive fractional systems.

\section{Concluding remarks}

The concept of cone fractional linear systems has been introduced. Necessary and sufficient conditions for fractional systems to be cone fractional ones were established. Sufficient conditions for the reachability of cone fractional linear systems were also given. The conditions were illustrated with an example of a linear cone fractional system. Following (Kaczorek, 2007d), the results can be extended to the controllability to zero for cone fractional linear systems.

\section{Acknowledgment}

This work was supported by the Polish Ministry of Science and Higher Education under the grant no. NN 514 193933

\section{References}

Engheta, N. (1997). On the role of fractional calculus in electromagnetic theory, IEEE Transactions on Antennas and Propagation 39(4): 35-46.

Farina, L. and Rinaldi, S. (2000). Positive Linear Systems: Theory and Applications, J. Wiley, New York, NY.

Ferreira, N.M.F. and Machado, J.A.T. (2003). Fractional-order hybrid control of robotic manipulators, Proceedings of the 11-th International Conference on Advanced Robotics, ICAR'2003, Coimbra, Portugal, pp. 393-398.

Gałkowski, K. (2005). Fractional polynomials and nD systems. Proceedings of the IEEE International Symposium on Circuits and Systems, ISCAS'2005, Kobe, Japan, CD-ROM.

Kaczorek, T. (2002). Positive $1 D$ and $2 D$ Systems, SpringerVerlag, London.

Kaczorek, T. (2006). Computation of realizations of discretetime cone systems, Bulletin of the Polish Academy of Sciences 54(3): 347-350.

Kaczorek, T. (2007a). Reachability and controllability to zero tests for standard and positive fractional discrete-time systems, JESA Journal, 2007, (submitted).

Kaczorek, T. (2007b). Reachability and controllability to zero of positive fractional discrete-time systems, Machine Intelligence and Robotic Control 6(4): 139-143.

Kaczorek, T. (2007c). Cone-realizations for multivariable continuous-me systems with delays, Advances in Systems Science and Applications 8(1): 25-34.

Kaczorek, T. (2007d). Reachability and controllability to zero of cone fractional linear systems, Archives of Control Sciences 17(3): 357-367. 
Kaczorek, T. (2008). Fractional positive continuous-time linear systems and their reachability, International Journal of Applied Mathematics and Computer Science 18(2):223-228.

Miller, K. S. and Ross, B. (1993). An Introduction to the Fractional Calculus and Fractional Differential Equations, Wiley, New York, NY.

Nishimoto, K. (1984). Fractional Calculus, Decartess Press, Koriama.

Oldham, K. B. and Spanier, J. (1974). The Fractional Calculus, Academic Press, New York, NY.

Ortigueira, M. D. (1997). Fractional discrete-time linear systems, Proceedings of the IEEE International Conference on Acoustics, Speech, and Signal Processing 97, Munich, Germany, pp. 2241-2244.

Ostalczyk, P. (2000). The non-integer difference of the discretetime function and its application to the control system synthesis, International Journal of Systems Science 31(12): 1551-1561.

Ostalczyk, P. (2004). Fractional-order backward difference equivalent forms. Part I-Horner's form, Proceedings of the 1-st IFAC Workshop on Fractional Differentation and Its Applications, FDA'04, Enseirb, Bordeaux, France, pp. 342-347.

Ostalczyk, P. (2004). Fractional-order backward difference equivalent forms. Part II-Polynomial form, Proceedings of the 1-st IFAC Workshop on Fractional Differentation and Its Applications, FDA'04, Enseirb, Bordeaux, France, pp. 348-353.

Oustaloup, A. (1993). Commande CRONE, Hermès, Paris.

Oustaloup, A. (1995). La dèrivation non entière. Hermès, Paris.

Podlubny, I. (1999). Fractional Differential Equations, Academic Press, San Diego, CA.

Podlubny, I., Dorcak, L. and Kostial, I. (1997). On fractional derivatives, fractional order systems and $P I^{\lambda} D^{\mu}$ controllers, Proceedings of the 36-th IEEE Conference on Decision and Control, San Diego, CA, USA, pp. 49854990.

Reyes-Melo, M.E., Martinez-Vega, J.J., Guerrero-Salazar C.A. and Ortiz-Mendez, U. (2004). Modelling and relaxation phenomena in organic dielectric materials. Application of differential and integral operators of fractional order, Journal of Optoelectronics and Advanced Materials 6(3): 1037-1043.

Riu, D., Retiére, N. and Ivanes, M. (2001). Turbine generator modeling by non-integer order systems, Proceedings of the IEEE International Conference on Electric Machines and Drives Conference, IEMDC 2001, Cambridge, MA, USA, pp. 185-187.
Samko, S. G., Kilbas, A.A. and Martichew, O.I. (1993). Fractional Integrals and Derivative. Theory and Applications, Gordon \& Breac, London.

Sierociuk, D. and Dzieliński, D. (2006). Fractional Kalman filter algorithm for the states, parameters and order of fractional system estimation, International Journal of Applied Mathematics and Computer Science 16(1): 129-140.

Sjöberg, M. and Kari, L. (2002). Non-linear behavior of a rubber isolator system using fractional derivatives, Vehicle System Dynamics 37(3): 217-236.

Vinagre, B. M., Monje, C. A. and Calderon, A.J. (2002). Fractional order systems and fractional order control actions, Lecture 3 IEEE CDC'02 TW\#2: Fractional Calculus Applications in Automatic Control and Robotics.

Vinagre, B. M. and Feliu, V. (2002). Modeling and control of dynamic system using fractional calculus: Application to electrochemical processes and flexible structures, Proceedings of the 41-st IEEE Conference on Decision and Control, Las Vegas, NV, USA, pp. 214-239.

Zaborowsky, V. and Meylaov, R. (2001). Informational network traffic model based on fractional calculus, Proceedings of International Conference on Info-tech and Info-net, ICII 2001, Beijing, China, Vol. 1, pp. 58-63.

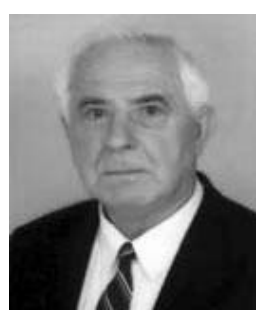

Tadeusz Kaczorek was born in 1932 in Poland. He received the M.Sc., Ph.D. and D.Sc. degrees from the Faculty of Electrical Engineering of the Warsaw University of Technology in 1956, 1962 and 1964, respectively. In the period 1968-69 he was the dean of the Faculty of Electrical Engineering, and in the period 1970-73 he was the deputy rector of the Warsaw University of Technology. Since 1974 he has been a full professor at the Warsaw University of Technology. In 1986 he was elected a corresponding member and in 1996 a full member of the Polish Academy of Sciences. In the period 1988-1991 he was the director of the Research Centre of the Polish Academy of Sciences in Rome. In June 1999 he was elected a full member of the Academy of Engineering in Poland. In 2004 he was elected a honorary member of the Hungarian Academy of Sciences. He has been awarded an honorary doctorate by several universities. His research interests cover systems theory and control theory, especially singular multidimensional systems, positive multidimensional systems, and singular positive 1D and 2D systems. He has published 21 books (six in English) and over 850 scientific papers.

Received: 21 April 2008 Revised: 8 June 2008 
